\title{
An Automated Negotiation-based Framework via Multi-Agent System for the Construction Domain
}

\author{
Moamin A. Mahmoud, Mohd Sharifuddin Ahmad, Mohd Zaliman M. Yusoff, and Arazi Idrus \\ Dept. of Computer Science and Information Technology, Universiti Tenaga Nasional (UNITEN), Malaysia
}

\begin{abstract}
In this paper, we propose an automated multi-agent negotiation framework for decision making in the construction domain. It enables software agents to conduct negotiations and autonomously make decisions. The proposed framework consists of two types of components, internal and external. Internal components are integrated into the agent architecture while the external components are blended within the environment to facilitate the negotiation process. The internal components are negotiation algorithm, negotiation style, negotiation protocol, and solution generators. The external components are the negotiation base and the conflict resolution algorithm. We also discuss the decision making process flow in such system. There are three main processes in decision making for specific projects, which are propose solutions, negotiate solutions and handling conflict outcomes (conflict resolution). We finally present the proposed architecture that enables software agents to conduct automated negotiation in the construction domain.
\end{abstract}

Keywords - Intelligent Software Agent, Multi-agent Systems, Agent and Negotiation, Automated Negotiation, Value Management, Construction Domain.

\section{INTRODUCTION}

In the construction domain, deciding on a new project is dependent upon a company's strategy. If the strategy is based on a decision made by a stakeholder, then it takes a very short time to decide. However, such decision has no value in terms of value management, because the decision-making process does not include other experienced stakeholders that hold different backgrounds.

Figure 1 considers a value management approach that emphasizes on involving various stakeholders in the decision-making process to arrive at a single valued solution. In other words, the various stakeholders with different backgrounds that have a stake in the project must contribute to the decision. In fact, these stakeholders often belong to different departments and possess different perspectives about the solutions according to their background and positions they hold.

For example, a project manager usually cares more about the cost of a project than the function while a design manager is more concerned about the function than the cost. Thus, for any decision to be made regarding a new project, stakeholders must propose a single optimal solution. However, a problem may arise when stakeholders propose more than one solution. In such situation, stakeholders need to negotiate on the proposed solutions and agree on a single solution. But the negotiation may not be easy and smooth because when stakeholders possess different backgrounds, often their views about the optimal solution for a particular project are different. Such differences cause conflicts in arriving at a decision. In addition, stakeholders may work at different branches throughout the country or other parts of the world which make a meeting for decision more difficult and costly. While applying Value Management on decision making in the construction domain is useful, it faces communication difficulties between stockholders and conflicting issues that require negotiation.

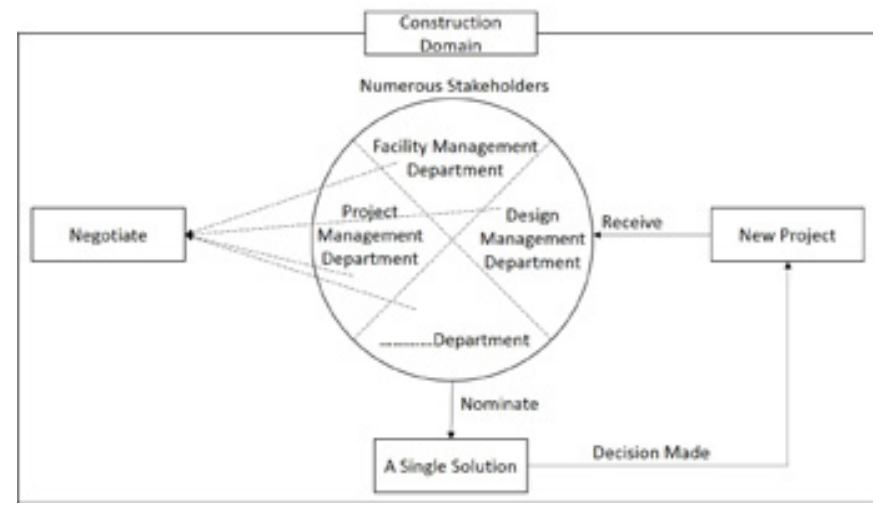

Figure 1 A Decision Making based on Value Management in Construction Domain

In this paper, we attempt to overcome these difficulties by proposing a Value-based Automated Negotiation Model utilizing the multi-agent system's approach. It enables software agents to conduct negotiations and autonomously arrive at a decision.

While this work is inspired by the work of Utomo [1], his study is only in conceptual level and lacks an intelligent agent architecture that aids an agent to interact with other agents and respond to its environment and eventually influences its autonomy level in decision making. Automated Negotiation as a very complicated system could not be efficiently used if agents have trivial architecture. Moreover, he does not incorporate the different negotiation styles to the agent architecture which could help the agents in mimicking humans' styles in negotiation. Briefly, the major development that we intend to do is to develop concrete agent architecture such as the Belief-Desir-Intention architecture and explore the potential components that an agent could employ to conduct useful and efficient negotiations. Consequently, we consult the various resources that are presented by Utomo [1] to come up with our framework.

The next section dwells upon the related work on automated negotiation. Section 3 presents the proposed framework. In Section 4, we discuss the decision making process flow. Section 5 presents the agent architecture and Section 6 concludes the paper.

\section{RELATED Work}

In this section, we discuss two prominent topics to this research which are, value management, and applications of negotiation in multiagent systems.

Value Management (VM) is defined as "a structured, organized team approach to identify the functions of a project, product, or service 
that will recognize techniques and provide the necessary functions to meet the required performance at the lowest overall cost" [17]. VM works on identifying and eliminating unnecessary cost [18] but without affecting a quality parameter [19]. VM is based on data collection method from reliable resources and functional requirements to fulfill the needs, wants and desires of the customers [1]. According to Kelly and Male [20], VM is a multidisciplinary, team-oriented approach to problem solving.

The application of VM in decision making has been reported by many researchers $[1,21,22]$. One of the techniques that is relevant to VM is weighting and scoring in which a decision needs to be made in selecting an option from a number of competing options, and the best option is not immediately identifiable [1, 23, 24].

Intelligent software agents have been widely used in distributed artificial intelligence and due to their autonomous, self-interested and rational abilities, agents are well-suited for automated negotiation on behalf of humans [2, 25, 26, 27, 28, 29, 30]. According to Kexing [2], automated negotiation is a system that applies artificial intelligence and information and communication technology to negotiation strategies, utilizing agent and decision theories.

Numerous research have discussed the negotiation on multi-agents systems in various domains $[3,4,5,6,7]$. Few of them study the issues of conflict resolution and negotiation in construction domain $[1,8,9]$. Anumba et al. [9] presented two main negotiation theories; mechanical and behavior theories. The mechanical theory is inspired by game theories which are mathematical models relied on rational behavior assumption, while the behavior theory studies human behavior in negotiation.

Coutinho et al. [10] proposed a negotiation framework to serve collaboration in enterprise networks to improve the sustainability of interoperability within enterprise information systems. Utomo [1] presented a conceptual model of automated negotiation that consists of methodology of negotiation and agent based negotiation. Dzeng and Lin [11] presented an agent-based system to support negotiation between construction and suppliers via the Internet. Anumba et al. [12] proposed a collaborative design of light industrial buildings based on multi-agent systems to automate the interaction and negotiation between the design members. Ren et al. [4] developed a multi-agent system representing participants, who negotiate with each other to resolve construction claims.

\section{A Conceptual Automated Negotiation Framework}

From our initial investigation of the literature $[1,2,3,4,8,9]$, we observe that agents need to be integrated with six main components to conduct negotiations, which are classified, in this research, into internal and external components. The internal components are negotiation algorithm, negotiation style, negotiation protocol, and solution generator. The components are integrated with a BDI agent architecture (as discussed in Section 5). The external components are the negotiation base and the conflict resolution algorithm.

As shown in Figure 2, the negotiation algorithm presents a formal and intelligent procedure that maintains negotiations with other agents. Each agent is endowed with a negotiation style that represents the agent's approach to negotiation. Each agent possesses one style, either competing style or collaborating style.

For agents to conduct negotiations systematically, they must have a negotiation protocol, which controls the negotiations process between agents. For example, agents could possibly negotiate individually or form groups (coalitions) before negotiating. They could also negotiate directly by sending messages to each other or deploy another method to share their inputs. Finally, agents must be able to generate solutions that conform to their interests and reap the benefits of negotiation.

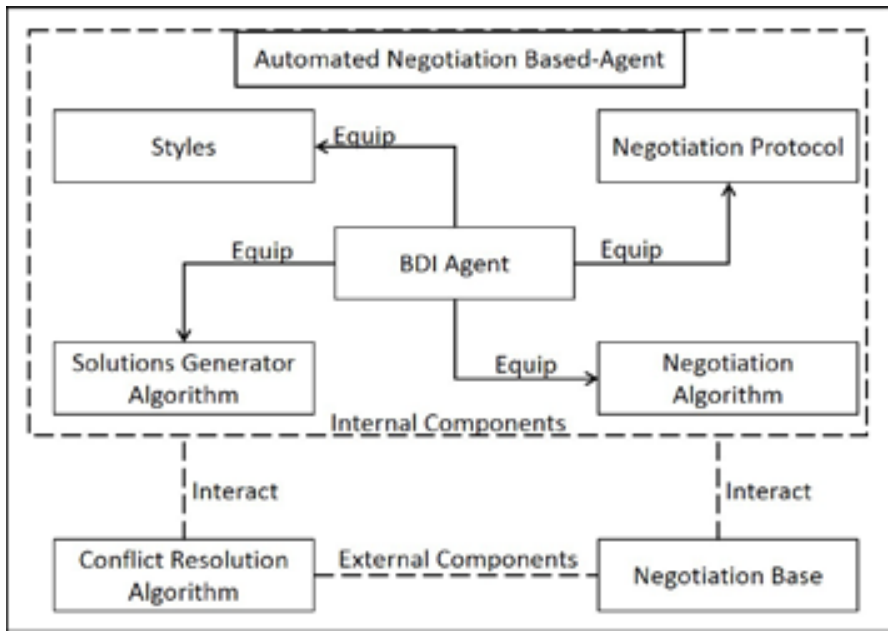

Figure 2. Automated Negotiation Model based on Multi-agent System (ANMAS)

The Negotiation Base represents the negotiation hub that contains suggested solutions of negotiations used by agents in sharing their solutions and form coalitions. The base reduces direct interactions between agents that would increase the network load.

The conflict resolution algorithm handles negotiations outcomes. If agents have not agreed on a single solution, the conflict resolution algorithm works on solving that conflict. Consequently, the proposed framework with the internal and external components manifests the Automated Negotiation Model based on the Multi-agent System (ANMAS).

\section{A. A Negotiation Styles}

According to Utomo [1], there are five main negotiation styles that constitute two types of outcomes, which are Competing, Avoiding, Collaborating, Accommodating, and Compromising, and the two outcomes are Cooperative and Assertiveness.

Accordingly, Each agent possess one style, and this style forms one negotiation outcome whether it is Cooperative or Assertiveness, e.g. agent a is Cooperative type and possess Accommodating style.

\section{B. Negotiation Protocol}

Agents conduct negotiation according to a predefined protocol. Such protocol ensures that the negotiation progresses smoothly.

\section{Solution Generator Algorithm}

For an agent to conduct negotiation, it should be able to propose solutions and rank them from 1st to nth solution for the next stage of the negotiation operation.

In real situations, various stakeholders have different level of interest about the cost and function parameters based on their positions and values they uphold. Thus, those stakeholders appraise their solutions based on their interest level on these parameters. For example, in the construction domain, a Design Manager cares more about the function in contrast with a Project Manager who cares more about the cost, while a Facility Manager's interest is in between the Design and Project Managers' interests. Therefore, the Design Manager normally attempts to find a solution that provides high function, whereas the Project Manager normally attempts to find a solution that provides low cost. The Facility Manager attempts to find a moderate solution that provides acceptable cost and function. 
Consequently, The Solutions Generator Algorithm will be inspired by the two main parameters of Value Management which are Cost and Function to deliver value solutions.

\section{Negotiation base}

The Negotiation Base represents the negotiation hub that is used by agents to form negotiations by sharing their solutions and form coalitions. The base helps in reducing direct interactions between agents that increase the network load. All negotiations are processed via this base which is accessible by all agents.

\section{E. Negotiation Algorithm}

The algorithm implements the negotiation process between agents. The process starts when each agent submits its solutions to the negotiation base. Each agent then reviews each solution's and accordingly sets a plan to conduct negotiation.

\section{F. Conflict Resolution Algorithm}

The need for this algorithm is based on the negotiation algorithm outcomes. Since any project needs a single solution, then when the negotiation algorithm outcome is a single solution, agents skip this algorithm. But when the outcome is several solutions, then another process is needed to resolve this conflict. Such situation represents a conflict between agents about the solution of that project.

\section{Decision Making Process Flow}

A decision made by agents goes through several processes. These processes work by gradually reducing candidate solutions of a project until a single solution is reached. Consequently, in this work, the process of nominating a single solution from a set of solutions is called decision making.

There are three main processes in decision making for a specific project, which are propose solutions, negotiate solutions and handling conflict outcomes (conflict resolution).

- Propose solutions: In this process, each agent proposes solutions and ranks them from 1 st to nth solution where $\mathrm{n}$ is any natural number.

- Negotiate solutions: When ranked solutions are ready, agents negotiate by submitting their ranked solutions to each other. Since each agent's target is to maximize its utility by selecting a solution that has a better order, each agent prepares a plan. Using these plans, agents form coalitions among them based on similar plans. These coalitions continuously compare plans with each other until a single or more solutions converge after exhausting all attempts.

- Resolve conflict: If agent coalitions agree upon a single solution, then this process is forfeited, but if there are two or more conflicting solutions, then the conflicts need to be resolved. This process resolves conflicts based on each coalition's strength and its solutions' risks. From these two parameters, this process drops solutions until a single solution is reached.

Figure 3 shows the decision making flowchart as described above. The process starts when agents receive a new project. The agents first propose solutions in ranked order. They then negotiate these solutions. If they agree upon a single solution, then the decision is made, otherwise, the conflict resolution process takes over to drop the weak and risky solutions. If the outcome of the conflict resolution process is a single solution then the decision is made. Otherwise, the agents negotiate the outcome of the conflict resolution process. Ultimately, one coalition's solution is accepted.

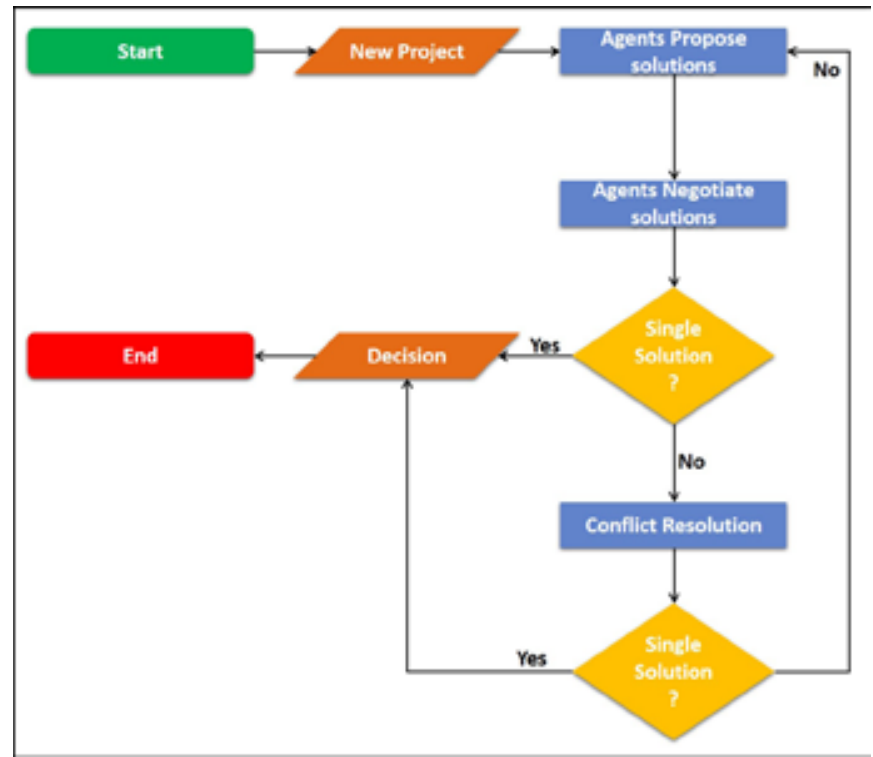

Figure 3 Decision Making Flowchart

\section{The Belief-Desire-Intention (BDI) Agent Architecture}

This section presents an architecture that enables software agents to mimic human behaviors and styles in building an automated negotiation system in the construction domain. In this work, we develop BDI agents that are widely used by researchers to build intelligent agents.

The BDI agent consists of three main components that are affected by the environment; Belief, Desire, and Intention. Agents usually perform tasks within an environment and they exploit the environment to update their goals. The agents' beliefs are influenced by the environmental changes. The belief in turn updates their desires and the intentions.

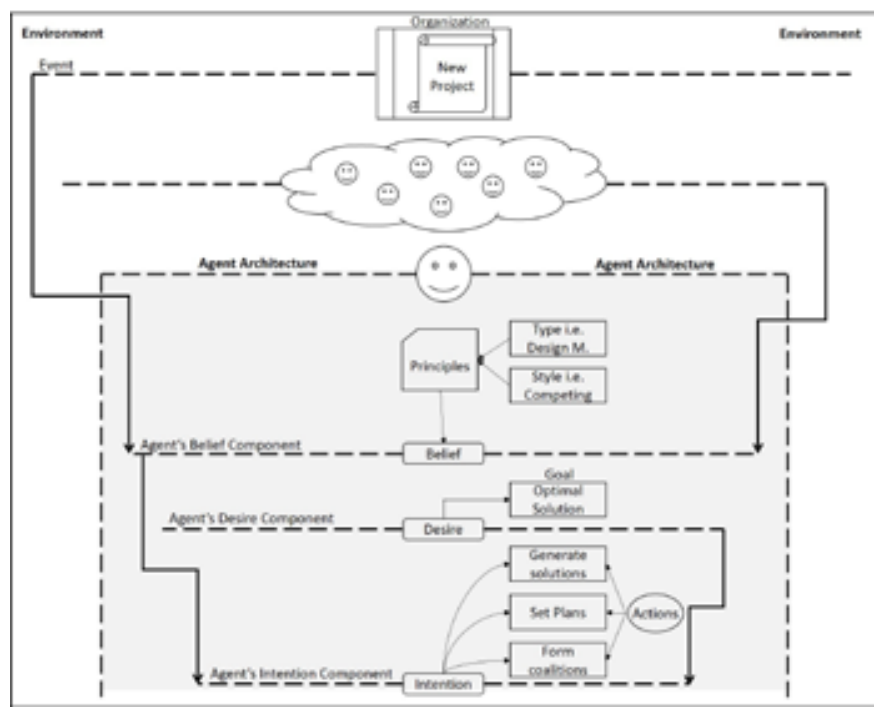

Figure 4. The Proposed Agent Architecture

As shown in Figure 4, the proposed agent architecture consists of the outer area that represents the environment and the inner area that represents the decision making process. The environment constitutes the variables of new project information and agent's activities information, e.g. interactions, decisions, negotiations, coalitions. The belief component within the architecture is influenced by the environment and agent attributes, which include the agent type, e.g. 
Design Manager, and the agent style e.g. competing. The desire component represents an agent's goal.

In the construction domain, each agent attempts to ensure its first rank solution wins. If it is not possible, it works on the second rank and so on. This scenario represents its desire or goal. From the agent's belief and desire, it performs actions which represent the intention components. The intention component represents a bridge between the belief and the desire, in other words, it represents the practical steps to achieve the desire according to its belief about the environment and the attributes.

\section{CONCLUSION AND FutURE WORK}

To create a multi-agent automated negotiation model, agents need to be integrated with several components. In this paper, we identify four internal components (negotiation algorithm, negotiation style, negotiation protocol, and solution generators) integrated with the agent design and two external components (the negotiation base and the conflict resolution algorithm) within the environment. These components constitute the proposed framework.

We also discuss the decision making process flow in such system, consisting of three main processes which are propose solutions, negotiate solutions and handling conflict outcomes (conflict resolution). We finally reveal our proposed agent's architecture to conduct automated negotiation in the construction domain.

Since this work is in its theoretical stage, it only presents the conceptual underpinnings of pertinent issues in negotiation and does not present the experimental results. Such outcome will be presented in our future work.

In addition, for our future work, we shall study and propose mechanisms for the three methods needed by the decision making process which are Agent Proposes Solutions, Agent Negotiate Solutions and Conflict Resolution.

\section{REFERENCES}

[1] Utomo C., Development of a negotiation Support Model for Value Management in Construction, $\mathrm{PhD}$ Thesis, University Teknologi PETRONAS, December 2009.

[2] Kexing L.. A survey of agent based automated negotiation. In Network Computing and Information Security (NCIS), 2011 International Conference on, vol. 2, pp. 24-27 (IEEE, 2011).

[3] Beer M., d'Inverno M., Jennings R.N., Luck M., Preist C., Schroeder M., Negotiation in multi-agent systems Knowledge Engineering Review, 14 (3) (1999), pp. 285-289

[4] Z. Ren, C.J. Anumba, Multi-agent systems in construction - state of the art and prospects, Automation in Construction, 13 (2004), pp. 421-434

[5] M. Wang, H. Wang, D. Vogel, K. Kumar, D.K.W. Chiu Agent-based negotiation and decision making for dynamic supply chain formation Engineering Applications of Artificial Intelligence, 22 (7) (2009), pp. 1046-1055

[6] Utomo C., Idrus A., A Concept toward Negotiation Support for Value Management on Sustainable Construction, Journal of Sustainable Development Vol 4, No 6 (2011).

[7] Victor Sanchez-Anguix , Vicente Julian , Vicente Botti , Ana GarcíaFornes, Tasks for agent-based negotiation teams: Analysis, review, and challenges, Engineering Applications of Artificial Intelligence, v.26 n.10, p.2480-2494, November, 2013.

[8] Anderson, R. M., Hobbs, B. F., \& Bell, M. L. (2002). Multi-objective decision-making in negotiation and conflict resolution. Encyclopedia of Life Support Systems. New York: Eolss Publishers.,

[9] C. J. Anumba, O. O. Ugwu, Z. Ren, Agents And Multi-agent Systems In Construction, Spon Press, 2005.

[10] Coutinho, C., Cretant, A., Ferreira da Silva, C., Ghodous, P., \& Jardim-Goncalves, R. (2014). Service-based negotiation for advanced collaboration in enterprise networks. Journal of Intelligent Manufacturing. doi:10.1007/s10845-013-0857-4.

[11] Dzeng, R. J., \& Lin, Y. C. (2004). Intelligent agents for supporting construction procurement negotiation. Expert Systems with Applications, 27(1), 107-119.

[12] C.J. Anumba, Z. Ren, A. Thorpe, O.O. Ugwu, L. Newnham, Negotiation within a multi-agent system for the collaborative design of light industrial buildings Adv Eng Software, 34 (7) (2003), pp. 389-401

[13] Johnson, H., Johnson, P., Task Knowledge Structures: Psychological basis and integration into system design, Acta Psychologica, Vol. 78, 1991, pp. 3-26.

[14] Friend, M. and Cook, L. The new Mainstreeem. Instructor 30-36.

[15] Matthews , J. Implications for collaborative educators preparation and development: A sample instructional approach in Diana G Pounder (Editor) Restructuring School of Collaboration: Promise and Pitfalls, State University of NewYwork Press. 1998

[16] Holley, W. H. and Jennings K.M. The labour relations process. 8th ed., Chapter 6. Mason, OH: Thomsan/South-Westeren, 2005.

[17] SAVE International, value methodology standards, 2001.

[18] Kelly J. and Male S., Value Management. Chapter 5 in Kelly J., Morledge R. and Wilkinson S (eds.) Best Value in Construction Oxford UK, Blackwell, pp 77-99, 2002.

[19] Mukhopadhyaya A.K., Value Engineering Concept, Techniques and Applications, Response Books, New Delhi, 2003.

[20] Kelly J. and Male S., Value Management in Decision and Construction, The Economic Management of Projects. Spon Press, London.

[21] Jaapar, A., Endut, I.R., Bari, N.A.A. and Takim, R. The impact of value management implementation in Malaysia. Journal of Sustainable Development 2 (2), 2009.

[22] Shen, Q., Chung, J.K.H., Li, H. and Shen, L.. A Group Support System for improving value management studies in construction. Automation in Construction, 13 (2004): 209-224, 2004.

[23] Cariaga, I, El-Diraby, T and Osman, H., Integrating Value Analysis and Quality Function Deployment for Evaluating Design Alternatives. Construction Engineering and Management, 133(10), 761-770, 2007.

[24] Qing Y. and Wanhua Q., 2007, Value Engineering Analysis and Evaluation. For the Second Beijing Capital Airport. Value World, Spring, SAVE International.

[25] Ahmed M., Ahmad M S, Yusoff M Z M, Modeling Agent-based Collaborative Process, The 2nd International Conference on Computational Collective Intelligence Technology and Applications (ICCCI 2010), pp. 296-305, ISBN:3-642-16692-X 978-3-642-16692-1, 10-12 November, 2010 Taiwan.

[26] Moamin A. Mahmoud, Mohd Sharifuddin Ahmad, Mohd Zaliman M. Yusoff, and Arazi Idrus. "Automated Multi-agent Negotiation Framework for the Construction Domain." Distributed Computing and Artificial Intelligence, (DCAI' 15), Spain, 3th-5th June, 2015. In Springer's Advances in Intelligent Systems and Computing, Springer International Publishing, Volume 373, 2015, pp 203-210.

[27] Itaiwi A. K., Ahmad M. S., Hamid N. H. A., Jaafar N. H., Mahmoud M. A., A Framework for Resolving Task Overload Problems Using Intelligent Software Agents, 2011 IEEE International Conference on Control System, Computing and Engineering, ICCSCE11,2011.

[28] Ahmed M., Ahmad M. S., and Yusoff M. Z. M., "A Collaborative Framework for Multiagent Systems." International Journal of Agent Technologies and Systems (IJATS), 3(4):1-18, 2011.

[29] Ahmed M., Ahmad M. S., and Yusoff M. Z. M.,, Mitigating Human-Human Collaboration Problems using Software Agents, The 4th International KES Symposium on Agents and Multi-Agent Systems - Technologies and Application (AMSTA 2010), pp. 203-212, ISBN:3-642-13479-3 978-3642-13479-1, Gdynia, Poland, 23 - 25 June 2010.

[30] Al-Mutazbellah Khamees Itaiwi, Mohd Sharifuddin Ahmad, Nurzeatul Hamimah Abd Hamid, Nur Huda Jaafar, Moamin A. Mahmoud, A Multi-agent Framework for Dynamic Task Assignment and Delegation in Workload Distribution, International Conference on Computer \& Information Sciences, 12 June 2012. 


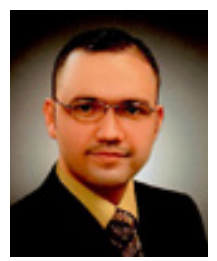

Moamin A. Mahmoud obtained his Bachelor in Mathematics from the College of Mathematics and Computer Science, University of Mosul, Iraq in 2007. $\mathrm{He}$ obtained his Master of Information Technology at the College of Graduate Studies, Universiti Tenaga Nasional (UNITEN), Malaysia in 2010, and PhD of Information and Communication Technology from Universiti Tenaga Nasional (UNITEN), Malaysia in 2013. His research interests are in the area of software agents, agent behavior in open societies, and social sciences simulation.

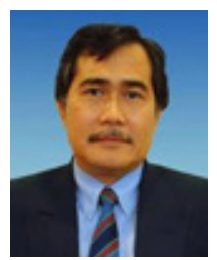

Mohd Sharifuddin. Ahmad received his B.Sc. in Electrical and Electronic Engineering from Brighton Polytechnic, UK in 1980. He started his career as a power plant engineer specialising in Process Instrumentation and Control in 1980. After completing his MSc in Artificial Intelligence from Cranfield University, UK in 1995, he joined UNITEN as a Principal Lecturer and Head of Dept. of Computer Science and Information Technology. He obtained his $\mathrm{PhD}$ from Imperial College, London, UK in 2005. He has been an associate professor at UNITEN since 2006. His research interests include applying constraints to develop collaborative frameworks in multi-agent systems, collaborative interactions in multi-agent systems and tacit knowledge management using AI techniques.

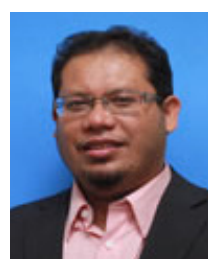

Mohd Zaliman Mohd Yusoff obtained his Msc and Phd in Computer Science from Universiti Kebangsaan Malaysia in 1998 and 2013 respectively. He started his career as a Lecturer at UNITEN in 1998 and has been appointed as a Principle Lecturer at UNITEN since 2008. His has produced and presented about 86 papers for local and international conferences. His research interest includes modeling and applying emotions in various domains including educational systems, norms based system including detection and assimilation strategies using software agents achitecture. He is also interested in modeling agent's trust and reputation in computer forensic and knowledge discovery systems. He is an active member of IEEE (91279801) and serves as the exco committee of Computer Society Malaysia Chapter since 2013.

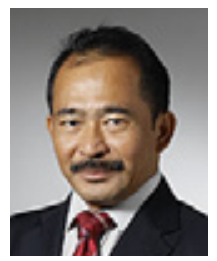

AraziIdrusB.Eng.(Hons)inCivilandStructuralEngineering (Sheffield University, UK), M.Sc. (Cranfield University, UK), Ph.D (Imperial College, University of London, UK). Field of Specialization: Project Management, Construction It, Construction Productivity, Value Management; PPP/PFI, IBS Construction, Building Maintenance Management, Risk-Based Assessment, Concrete Repairs,Blast Design, and Load Resistance Factor Design of Offshore Structures 